\title{
Application of Multigroup Technology in Non-Small-Cell Lung Cancer with Qi Stagnation and Blood Stasis Syndrome
}

\author{
Guan-Jun Ma $\mathbb{D}^{1},{ }^{1}$ Xiang Qian, ${ }^{2,3}$ Zhuo Chen, ${ }^{2,3}$ Sha-Sha Chen, ${ }^{4}$ and Ai-Qin Zhang $\mathbb{D}^{2,3}$ \\ ${ }^{1}$ Department of Geriatric Oncology, Affiliated Hangzhou Cancer Hospital, Zhejiang University School of Medicine, \\ 34 Yanguan Lane, Hangzhou 310002, China \\ ${ }^{2}$ Department of Traditional Chinese Medicine, \\ The Cancer Hospital of the University of Chinese Academy of Sciences (Zhejiang Cancer Hospital), 1 Banshandong Road, \\ Hangzhou, Zhejiang 310022, China \\ ${ }^{3}$ Institute of Basic Medicine and Cancer (IBMC), Chinese Academy of Sciences, 1 Banshandong Road, Hangzhou, \\ Zhejiang 310022, China \\ ${ }^{4}$ Department of Traditional Chinese Medicine, Taizhou Cancer Hospital, 50 Zhenxin Road, Wenling, Zhejiang 317502, China
}

Correspondence should be addressed to Ai-Qin Zhang; zhanghaojianbb@163.com

Received 24 April 2021; Accepted 29 June 2021; Published 9 July 2021

Academic Editor: Zhen Chen

Copyright ( 2021 Guan-Jun Ma et al. This is an open access article distributed under the Creative Commons Attribution License, which permits unrestricted use, distribution, and reproduction in any medium, provided the original work is properly cited.

Objective. To explore the basic characteristics of intestinal flora, metabolomics, and proteomics of non-small cell lung cancer (NSCLC) in patients with Qi stagnation and blood stasis syndrome. Methods. Twelve NSCLC patients with Qi stagnation and blood stasis syndrome were selected for the QZXY group and 15 healthy volunteers were selected for the control group. Fecal samples from the two groups were collected to evaluate intestinal microecology using the 16s rDNA technique. Serum samples were collected to compare the differences in metabolomics and proteomics between the two groups using liquid chromatographymass spectrometry (LC-MS). Another 34 NSCLC patients with other syndromes were selected for the nQZXY group and their serum samples were collected. Metabolomics differences between the QZXY and nQZXY groups were compared using LC-MS, and four metabolites with the most obvious differences were selected for receiver operation characteristic curve representation. Finally, multigroup results were analyzed using the WGCNA software. Results. There were two significantly different types of bacteria (Aerococcaceae and Abiotrophia), 11 different proteins (six upregulated and five downregulated), and 38 different metabolites (nine upregulated, 29 downregulated) between the QZXY and control groups. There was a correlation between differential bacteria, proteins, and metabolites. The conjoint analysis found that the different substances were related to MAPK, PI3K/Akt, Ras signaling pathway, cancer pathways, and cytokine-cytokine receptor interaction. There were four significant differences in metabolites (Pseudouridine, phenlacetyl-COA, L-glutamic, and phospho-anandamide) between the QZXY and nQZXY groups. Conclusions. NSCLC with Qi stagnation and blood stasis syndrome had specific intestinal flora and protein and metabolites, which were closely related to the occurrence and development of tumors.

\section{Introduction}

Lung cancer ranks first in the incidence and mortality rates of malignant tumors in China. Many studies [1-3] have shown that the application of traditional Chinese medicine (TCM) can control tumor progression, improve quality of life, and prolong survival time during treatment of nonsmall cell lung cancer (NSCLC). TCM may be a strong therapeutic candidate for tumors. Syndrome differentiation is one of the important principles of TCM targeted at recognizing and dealing with diseases. It is based on a comprehensive analysis of clinical information collected using the main TCM diagnosis procedures: observation, listening, questioning, and pulse analyses. In recent years, research on the standardization, objectification, and quantification of TCM syndromes has become popular.

Systems biology is an emerging discipline based on the development of modern and continuous biological 
experiment technology. Its research methods include transcriptomics, proteomics, metabolomics, and intestinal microecology. The concept of the system is similar to the holistic concept of TCM. TCM syndromes describe the functional state of the body at a certain stage as part of TCM theory. It is a high level generalization of the physiological and pathological state at a certain stage during the occurrence and evolution of the disease. The syndromes can be classified as instantaneous, dynamic, and integral.

Using systems biology methods to objectively study TCM syndromes is conducive to revealing their essence. The TCM pathological properties of lung cancer are as follows: deficiency in origin and excess in excess, the healthy Qi hurts first, and the evil Qi takes the opportunity to enter the lung. Qi stagnation and blood stasis are important pathogenesis processes of lung cancer [4] and [5], and the prescription of regulating Qi and activating blood has a good curative effect [6]. In this study, 16S rDNA and liquid chromatographymass spectrometry (LC-MS) technology were used to study the proteomics, metabolomics, and intestinal microecology of NSCLC with Qi stagnation and blood stasis syndrome, aiming to provide a biological basis for the diagnosis of TCM syndromes in NSCLC, and an experimental basis for the future of anticancer treatment using Chinese medicine.

\section{Materials}

2.1. General Information. The subjects of this study were 46 patients from the thoracic tumor surgery outpatient department of Zhejiang Cancer Hospital between March 1, 2019, and September 30, 2019, who planned to undergo radical resection of lung cancer without any treatment before enrollment. Twelve patients were identified to have the QZXY syndrome by two vice directors or TCM physicians at the same time. The remaining 34 patients were identified as participants with other syndromes (nine patients had spleen deficiency and dampness stagnation (PXSZ) syndrome, 11 patients had a deficiency of Qi and Yin (QYLX) syndrome, 14 patients had Yin deficiency with internal heat (YXNR) syndrome). In addition, 15 healthy volunteers were included.

This clinical study was approved by the Ethics Committee of Zhejiang Cancer Hospital (Approval no. IRB-2018219).

\subsection{Diagnostic Criteria of the Patients}

The patients were confirmed to have NSCLC by histopathology and/or cytopathology [7].

The patients met the following diagnostic criteria of the QZXY syndrome $[8,9]$, which included a cough that was not smooth, shortness of breath, chest pain as cone or thorns, dry mouth, constipation, hemoptysis or phlegm that is bloody and dark red, lip dark purple tongue, tongue ecchymosis, thin yellow moss, and pulse string or astringent.

\subsection{Inclusion Criteria of Patients}

\subsubsection{NSCLC Patients}

Patients meet the diagnostic criteria

Patients had no history of other malignancies

Patient ages ranged from 18 to 75 years

Patients signed the informed consent form in person

\subsubsection{Volunteers}

Volunteers had no history of malignancies or major diseases, such as cardiovascular, cerebrovascular, liver, kidney, and endocrine diseases

Volunteer ages ranged from 18 to 75 years

Volunteers signed the informed consent form in person

\subsubsection{Exclusion Criteria of Patients}

Patients were mentally ill and incapacitated

Patients or volunteers had been treated with antibiotics for nearly 3 weeks

\section{Methods}

3.1. Intestinal Microecological Analysis. Fecal samples (200 mg each) were mixed with $30 \mathrm{~mL}$ of buffer solution. After centrifugation at $1,000 \mathrm{rpm}$ for $5 \mathrm{~min}$, the precipitation was collected and resuspended in $10 \mathrm{~mL}$ of buffer solution. Fecal microbial DNA was extracted using the QIAAMP Fast DNA Stool Mini Kit. After extraction, the total DNA was inspected using agarose gel electrophoresis and Thermo Nanodrop 2000 UV microspectrophotometer. The samples were amplified in 16S V3-V4 region. The primer information was as follows: forward primer: CCTACGGGNGGCWGCAG; reverse primer: GACTACHVGGGTATCTAATCC. The diluted genomic DNA was used as a template for PCR using Phanta Max Master Mix (Vazyme) high fidelity enzyme. The reaction conditions of PCR were as follows: predenaturation at $95^{\circ} \mathrm{C}$ for $3^{\circ} \mathrm{min} ; 95^{\circ} \mathrm{C}$ denaturation for $30 \mathrm{~s}, 55^{\circ} \mathrm{C}$ annealing for $30 \mathrm{~s}$, 72 extension for $45 \mathrm{~s}$, and repeat 25 cycles. The final incubation was carried out at $72^{\circ} \mathrm{C}$ for $5 \mathrm{~min}$. The PCR reaction system included the following: $25 \mu \mathrm{L}$ of $2 \times$ Phanta Max Master Mix (Vazyme), $2 \mu \mathrm{L}$ of each primer $(10 \mu \mathrm{M})$, and DDH2O added to make up a total of $50 \mu \mathrm{L}$. PCR product $(1.5 \mu \mathrm{L})$ was analyzed using $120 \mathrm{~V}$ continuous electrophoresis for $20 \mathrm{~min}$ in $2 \%$ agarose gel. Subsequent UV imaging was performed using the gel imaging system.

After the quality inspection of the library, Qubit was used to quantify the concentration of the mixed library pool, Mixing was carried out according to the volume requirements of each sample. Finally, the Miseq sequencing program was run. Clean reads with the same sequence were sorted according to their abundance, while singletons were filtered out. USearch was used for clustering at a similarity degree of 0.97 . After chimeric filtering of the clustered 
sequences, OTU for species classification was obtained. Finally, all clean reads were matched to the OTU sequence. Reads that could be matched to the OTU were extracted to obtain the final mapped reads.

3.2. Proteomic Analysis. A total of $30 \mu \mathrm{g}$ of protein extract were mixed with $200 \mu \mathrm{L}$ of $8 \mathrm{M}$ urea. The mixture was centrifuged at $20^{\circ} \mathrm{C}$ and $14000 \mathrm{R}$ for $15 \mathrm{~min}$. The concentrate was diluted with $200 \mu \mathrm{L}$ of $8 \mathrm{M}$ urea in $0.1 \mathrm{M}$ Tris- $\mathrm{HCl}$ at $\mathrm{pH}$ 8.5 and centrifuged. Precipitates were diluted with $100 \mu \mathrm{L}$ of $8 \mathrm{M}$ urea in $0.1 \mathrm{M}$ Tris- $\mathrm{HCl}$ at $\mathrm{pH} 8.5$ and centrifuged again. The concentrate was digested in a wet chamber at $37^{\circ} \mathrm{C}$ for $12 \mathrm{~h}$ with trypsin (enzymatic to protein ratio: $1: 100,50 \mathrm{M}$ $\mathrm{MABC}$ ) and then centrifuged. The digestive solution was collected and rinsed with $50 \mu \mathrm{L}$ of $0.5 \mathrm{M}$ sodium chloride. The resulting solution was combined with $10 \%$ trifluoroacetic acid, acidified, and then desalted using C18Tips (Pierce, Thermo Science Titi C). Activation, equalization, peptide elution, and elution were performed according to the manufacturer's instructions. TIPS were activated with two rinses in 50\% acetonitrile (ACN) and equilibrated with two rinses in $0.1 \%$ trifluoroacetic acid (TFA) in water. The peptide-rich solution was slowly aspirated and distributed using TIP for 10 cycles. Two washing desalination processes were performed with $0.1 \%$ TFA. The desalinated peptide was eluted with $50 \mu \mathrm{L}$ of $50 \%$ can. The eluted product was dried in a vacuum and then suspended in $30 \mu \mathrm{L}$ of $0.1 \%$ formic acid. The solution containing the desalinized peptide $(3 \mu \mathrm{L})$ was separated using an EASY-Nano-LC system (Thermo Fisher Scientific) and online nanomobile liquid chromatography. The LC was connected to a $2 \mathrm{~cm}$ precolumn with an inner diameter of $100 \mu \mathrm{m}$ and filled with $5 \mu \mathrm{m}$ of $\mathrm{C} 18$ resin (Thermo Fisher Scientific). After the precolumn reaction, the $75 \mu \mathrm{m} \times 15 \mathrm{~cm}$ capillary column was filled with $3 \mu \mathrm{m}$ of $\mathrm{C} 18$ resin (Thermo Fisher Scientific). The polypeptides were eluted from the column front and analyzed on the column using step-by-step gradient elution, followed by a search of all the original files against the UniProt human protein sequence database in MaxQuant (version 1.6). Perseus was used to calculate the folding changes of each subgroup. Proteomes with more than twofold changes in the data frame $(>2$ or $<0.5)$ were labeled as significantly altered proteins.

3.3. Metabolomics Analysis. The serum sample $(200 \mu \mathrm{L})$ was placed on ice at room temperature. Then, $600 \mu \mathrm{L}$ of methanol/ethyl alcohol (1:1), which had been refrigerated overnight at $-20^{\circ} \mathrm{C}$, was added, swirled for $30 \mathrm{~s}$, and centrifuged at $14,000 \mathrm{rpm}$ at $4^{\circ} \mathrm{C}$ for $15 \mathrm{~min}$. The supernatant was placed into a centrifuge tube and dried with a vacuum centrifuge concentrator. After drying, the samples were redissolved in acetonitrile/methanol (80/20) and water mixture $(1: 1)$, swirled for $60 \mathrm{~s}$, and centrifuged at $14,000 \mathrm{rpm}$ at $4^{\circ} \mathrm{C}$ for $15 \mathrm{~min}$, and $4 \mu \mathrm{L}$ of the supernatant was extracted and analyzed using UPLC-Q/TOF-MS. The samples were separated on an ExionLC AD ultra-performance liquid chromatography (UHPLC) ACQUITY UPLC HSS T3 column $(100 \times 2.1 \mathrm{~mm}, 1.8 \mu \mathrm{m})$ using the following specifications: temperature of sample plate: $4^{\circ} \mathrm{C}$; column temperature: $40^{\circ} \mathrm{C}$; sample size: $4 \mu \mathrm{L}$; mobile phase composition $\mathrm{A}$ : acetonitrile (containing $0.1 \%$ formic acid), B: water (containing $0.1 \%$ formic acid); and flow rate: $0.3 \mathrm{~mL} /$ min. The elution gradient was $0-1 \mathrm{~min}, 95 \% \mathrm{~B} ; 1-20 \mathrm{~min}$, 95 1\%B; and 20-23 min, 1\%B. The samples were separated using UHPLC and analyzed with X-500R mass spectrometer (AB SCIEX). The ions with significant differences were screened using single- and multidimensional statistical analysis $(P<0.05$, FC $>1.5$ and VIP $>1.0)$ as potential biomarkers.

3.4. Combined Analysis of Intestinal Microecology and Metabolomics. Spearman's correlation analysis was performed on the secondary differential metabolites screened using metabolomics and significant differential level bacteria obtained via $16 \mathrm{~S}$ sequencing analysis in order to identify the relationship between microflora and metabolites. Based on the calculation results, appropriate screening conditions were selected to obtain the final correlation and network diagram.

3.5. Combined Proteomics-Metabolomics Analysis. By combining metabolomics and proteomics data using the KEGG pathway analysis, proteins and metabolites that are involved in significant changes in the same biological process (KEGG pathway) can be identified and used to quickly target key genes. This process was divided into the following steps: (1) check whether proteome and metabolome are related through KEGG metabolic pathway; (2) data screening for significant difference data and regulatory relationship; (3) GO and KEGG enrichment analysis; (4) clustering of expression patterns for linked data; and (5) creation of linked data network diagram.

3.6. Statistical Method. The intestinal floras with significant differences among different groups were identified using a rank-sum test. The threshold value for significance screening was a $P$ value $<0.05$. All $p$ values were verified using FDR. In the analysis of differential proteins, the differentially expressed protein needed to meet the following conditions: the coincidence difference ratio was 1.2 times (up-down) and $P$ value $<0.05$. The $t$-test method was limited to the comparison between two groups of samples with biological duplicates. When conducting differential metabolite analysis between two groups of samples, univariate analysis can intuitively show the significance of metabolite changes between two samples, thus helping to screen for potential marker metabolites (usually $\mathrm{FC}>1.5$ and $P$ value $<0.05$ as the screening criteria). Spearman's correlation coefficient was used for joint analysis. It is a linear correlation coefficient, which is a statistical quantity used to reflect the degree of linear correlation between two variables. The correlation coefficient is expressed using $r$, where $n$ is the sample size. It is the observed value and mean value of the two variables, respectively. $R$ describes the degree of linear correlation between two variables. The greater the absolute value of $R$, the stronger the correlation. The correlation analysis of 
significantly different metabolites between the QZXY and nQZXY groups was carried out using the binary logistic regression stepwise method. A receiver operating characteristic curve was used to evaluate the efficacy of differential metabolites in differentiating the two.

\section{Results}

4.1. Baseline Subject Data. Twelve NSCLC patients with QZXY syndrome were included in the QZXY group. A total of 34 NSCLC patients with other syndromes (PXSZ, QYLX, and YXNR) were included in the nQZXY group. Fifteen healthy volunteers formed the control group. The above information is presented in Table 1 .

\subsection{Differences between Microecological Groups}

4.2.1. Venn Diagram. The $16 \mathrm{~S}$ sequencing analysis showed that a total of 249 OTUs were generated from 27 samples (Figure 1).

4.2.2. Differential Species Analysis. Linear discriminant analysis (LDA) effect size (LEFSE) analysis used linear discriminant analysis (LDA) to estimate the influence of the abundance of each component (species) on the difference effect and to determine the communities or species that had a significantly different influence on sample division. The LDA scores obtained using linear regression analysis for the significant microbial groups are shown in Figure 2.

4.2.3. Analysis of Spearman's Correlation Coefficient in Different Species. LEFSE was used at each level or the ranksum test was used at the genus level (or at a specific level) to select the species with different abundance TOP30. Spearman's correlation heat map for species was created using the Corrplot package of $R$ software. The specific results are shown in Figure 3.

\subsection{Differences between Proteomics Groups}

4.3.1. Statistical Analysis of Upregulation and Downregulation of Differential Proteins. The up- and downregulation frequency statistics for differential proteins were used to determine the number of differential proteins under different experimental conditions. The horizontal axis represents the comparison group information, and the vertical axis represents the number of proteins. Red identifies the upregulated proteins, blue shows the downregulated proteins, and numbers represent the up- and downregulated proteins (Figure 4).

4.3.2. Functional Analysis of Differential Protein Genes. The term is the basic unit of GO analysis, and each term corresponds to an attribute. The GO significance enrichment analysis first mapped all of the differentially expressed genes
TABle 1: Sex, age, and stage data for the three groups.

\begin{tabular}{lcccccc}
\hline \multirow{2}{*}{ Group } & \multicolumn{2}{c}{ Gender } & \multirow{2}{*}{ Average age } & \multicolumn{3}{c}{ Clinical staging } \\
& Male & Female & & I & II & III \\
\hline QZXY & 8 & 4 & $59.25 \pm 9.60$ & 10 & 2 & 0 \\
Control & 7 & 8 & $61.40 \pm 7.02$ & 0 & 0 & 0 \\
nQZXY & 19 & 15 & $58.76 \pm 8.67$ & 29 & 3 & 2 \\
\hline
\end{tabular}

Statistical analysis indicated that the baseline data were comparable.

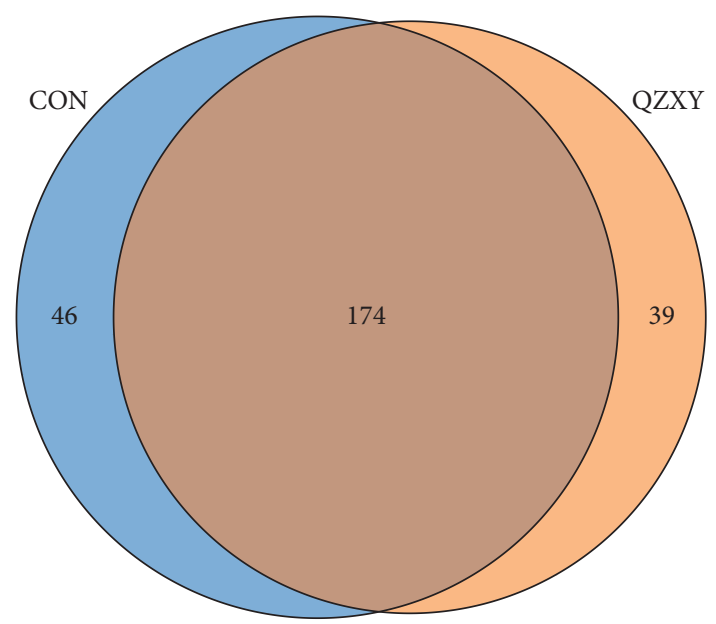

Figure 1: There were 174 common OTUs in the two groups. Compared to the intestinal flora in the control group, significant differences were present in the QZXY group. There were 39 unique OTUs in the QZXY group and 46 unique OTUs in the control group.

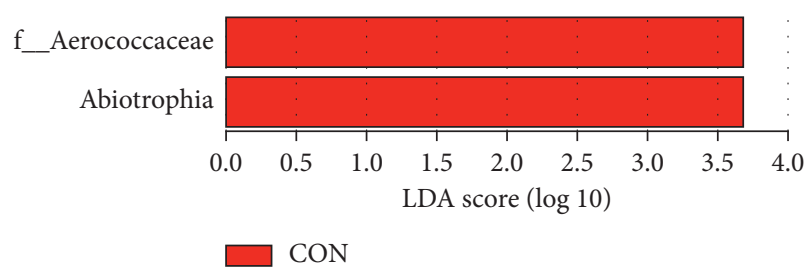

FIgURE 2: The bacterial genera with significant differences between the two groups $(P<0.05)$ were Aerococcaceae and Abiotrophia. The abundance level in the QZXY group was significantly lower than that in the control group.

to each term in the GO database, calculated the number of genes in each term, and then applied a hypergeometric test to identify the GO terms significantly enriched in the differentially expressed genes compared to the entire genome background. The results are shown in Figure 5.

\subsubsection{KEGG Pathway Analysis of Differential Protein.} The KEGG pathway was used as a unit of pathway enrichment analysis. The hypergeometric test was used to identify the pathways that were significantly enriched in the differentially expressed genes compared to the overall genome background. The results are shown in Figure 6. 


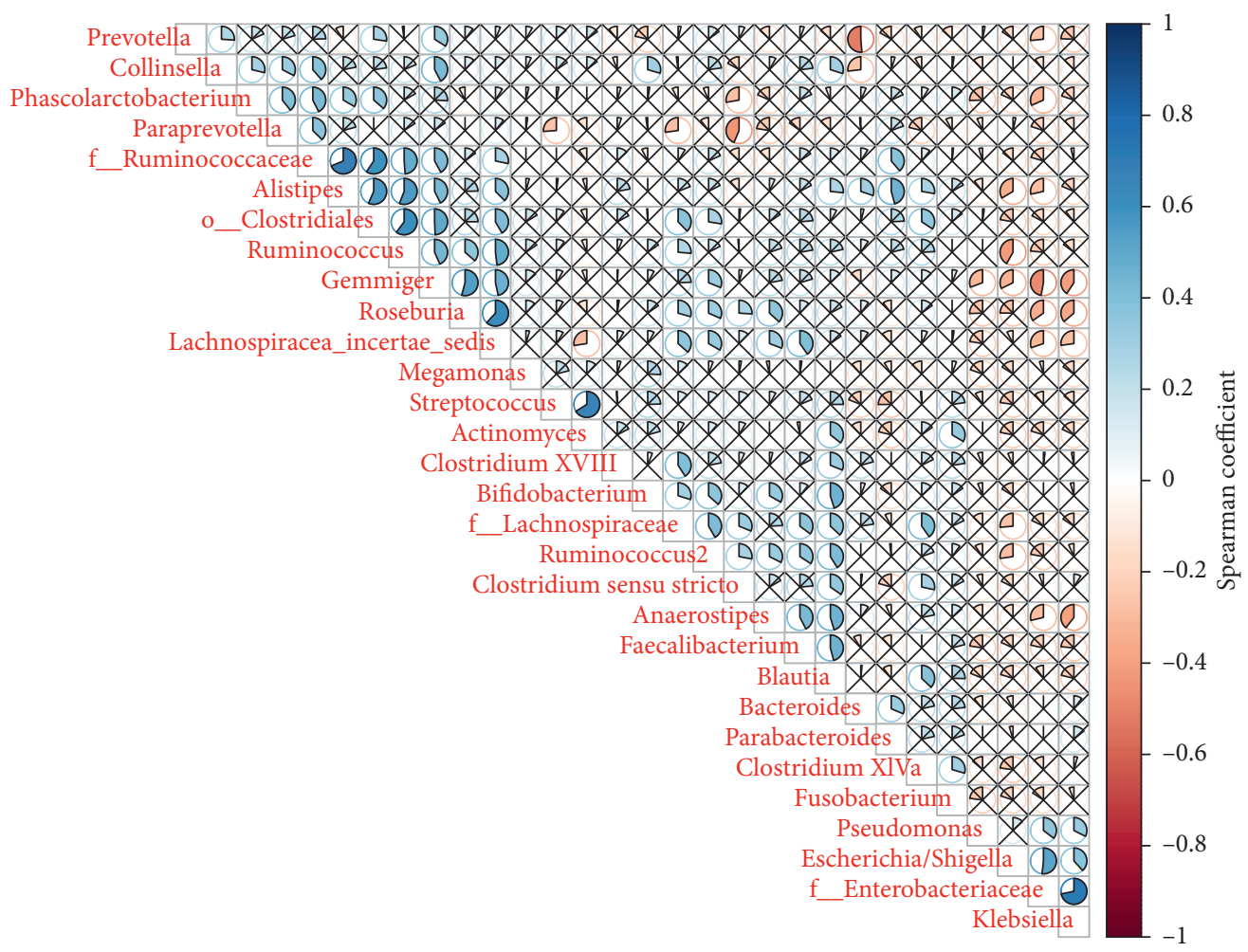

FIGURE 3: Spearman's correlation coefficient analysis of different species showed that there was a positive correlation between Roseburia and Lachnospiraceae incertae sedis. There was a positive correlation between Streptococcus and Actinomyces and between Enterobacteriaceae and Klebsiella.

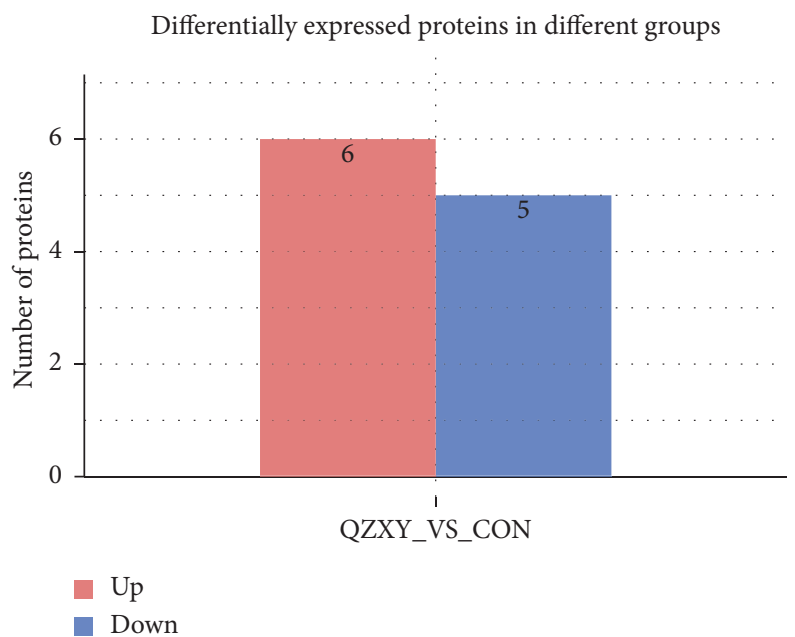

FIgURE 4: Compared to the control group, there were six upregulated proteins and five downregulated proteins in the QZXY group, for a total of 11 differential proteins.

4.4. Differences between Metabolomics Groups. In this study, univariate statistical analysis and multivariate statistical analysis were used to screen for significant difference metabolites. MetDNA and OSI/SMMS software were used for the structure identification of metabolites with significant differences. The specific identification results are shown in Table 2 and Figure 7.

The obtained differential metabolites were submitted to the Metaboanalyst 4.0 website for metabolic pathway analysis. The results are shown in Table 3. 


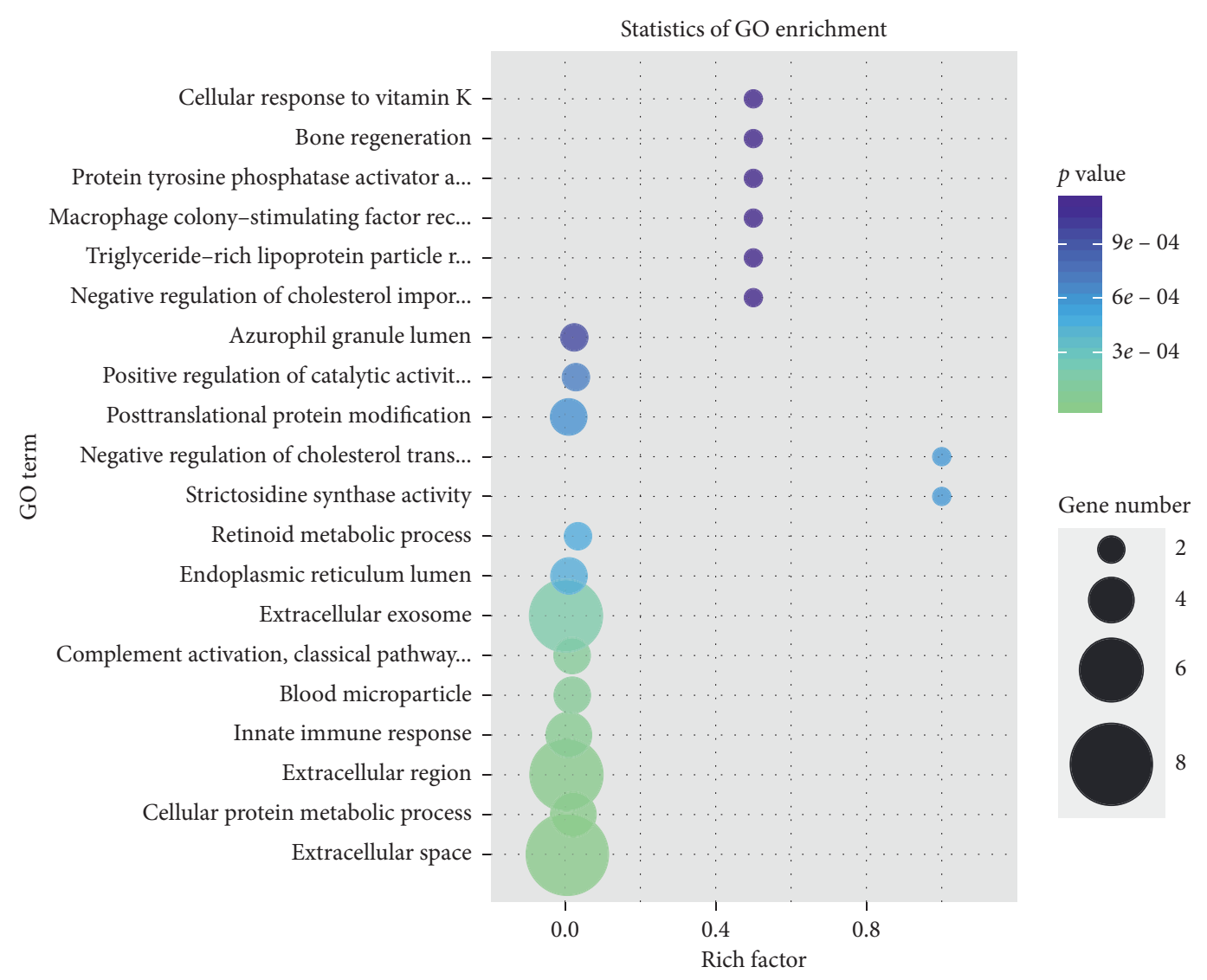

FIGURE 5: GO results showed that the proteomic genes in the QZXY group are extracellular space and extracellular exosome and can enhance the ability of metastasis and invasion of lung cancer cells and mediate tumor immunosuppression.

\subsection{Combined Analysis of Intestinal Microecology and Metabolomics}

4.5.1. Correlation Analysis of Differential Metabolites and Differential Flora. The combined analysis results of microflora and metabolites are shown in Figures 8 and 9, where red identifies a positive correlation and blue signifies a negative correlation.

Figures 8 and 9 show that Peptoniphilus was positively correlated with phenylacetyl-L-glutamine and indoxyl sulfate. Prevotella was negatively correlated with phenylacetylL-glutamine and 16-hydroxypalmitate. Bifidobacteria and 18-hydroxyoleate were also negatively correlated.

4.5.2. Differential Metabolites and Microflora Network Regulation Analysis. Different nodes in the figure represent different flora (round) or metabolites (triangular). The line between the flora and the metabolites represents the correlation between the two, where a solid line identifies a positive correlation and a dashed line represents a negative correlation. The results are shown in Figures 10 and 11.

4.5.3. Combined Proteomics-Metabolomics Analysis. The results of the combined proteomics-metabolite analysis are shown in Figure 12.
4.5.4. Metabolite Analysis of Difference between the QZXY Group and the nQZXY Group. ROC curves were used to analyze the different metabolites between the two groups. The results are shown in Figure 13.

\section{Discussion}

At present, the NSCLC treatment mainly involves different comprehensive treatment strategies according to pathological tumor types and clinical stages, which can somewhat improve the clinical symptoms and prolong survival time. However, individual differences lead to great variations in prognosis [10]. Increasing evidence [11, 12] has indicated that TCM with syndrome differentiation can improve the current situation. However, its validity has always been questioned because of the undefined molecular mechanism of syndromes. The present report attempted to provide a scientific explanation for the syndrome by observing the biological basis for the syndrome's type of Qi stagnation and blood stasis in NSCLC patients.

The present study found that peptone bacteria were positively correlated with glutamine, indolophenol, and other metabolites through network regulation analysis of differential metabolites and flora. The upregulated metabolite glutamine was found to inhibit the migration of human lung adenocarcinoma A549 cells, possibly by downregulating the expression of TNF- $\alpha$ and NF-KB p65 protein 


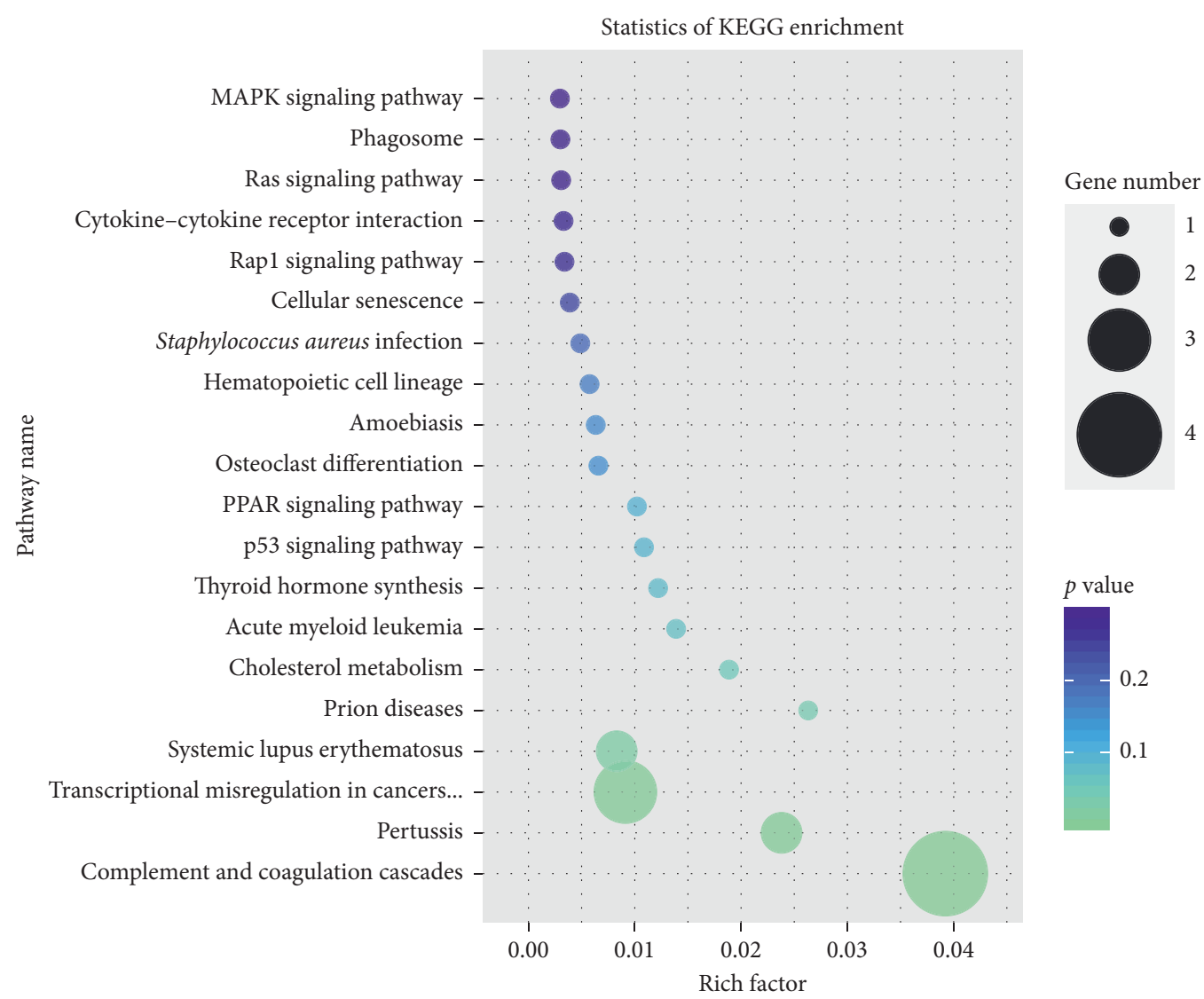

FIgURE 6: Pathways most associated with outcomes in the QZXY group included MAPK signaling pathway, Phagosome, Ras signaling pathway, and cytokine-cytokine receptor interaction. Tumor-related pathways were also analyzed.

TABLE 2: A total of 38 differential metabolites were identified in this study, including 14 metabolites in negative ion mode and 24 metabolites in positive ion mode.

\begin{tabular}{|c|c|c|c|c|c|c|c|c|}
\hline Number & $\begin{array}{c}\mathrm{RT} \\
(\mathrm{min})\end{array}$ & $\mathrm{m} / z(\mathrm{Da})$ & $P$ value & $\mathrm{FC}$ & VIP & Adduct & Formula & Compound name \\
\hline 1 & 4.09 & 212.0018 & 0.000752 & 0.31934 & 9.74487 & $\mathrm{M}-\mathrm{H}$ & $\mathrm{C}_{8} \mathrm{H}_{7} \mathrm{NO}_{4} \mathrm{~S}$ & Indoxyl sulfate \\
\hline 2 & 19.54 & 255.2324 & 0.011998 & 1.545 & 3.29746 & $\mathrm{M}-\mathrm{H}$ & $\mathrm{C}_{16} \mathrm{H}_{32} \mathrm{O}_{2}$ & Palmitic acid \\
\hline 3 & 4.08 & 263.1036 & 0.029296 & 0.50604 & 2.40863 & $\mathrm{M}-\mathrm{H}$ & $\mathrm{C}_{13} \mathrm{H}_{16} \mathrm{~N}_{2} \mathrm{O}_{4}$ & Phenylacetyl-L-glutamine \\
\hline 4 & 19.72 & 281.2472 & 0.018968 & 1.6259 & 8.45263 & $\mathrm{M}-\mathrm{H}$ & $\mathrm{C}_{18} \mathrm{H}_{34} \mathrm{O}_{2}$ & (11E)-Octadecenoic acid \\
\hline 5 & 17.53 & 277.2166 & 0.00647 & 1.7695 & 5.45746 & $\mathrm{M}-\mathrm{H}$ & $\mathrm{C}_{18} \mathrm{H}_{30} \mathrm{O}_{2}$ & $(6 \mathrm{Z}, 9 \mathrm{Z}, 12 \mathrm{Z})$-Octadecatrienoic acid \\
\hline 6 & 15.77 & 299.2594 & 0.011316 & 1.5535 & 1.449 & M-H & $\mathrm{C}_{18} \mathrm{H}_{36} \mathrm{O}_{3}$ & (R)-10-Hydroxystearate \\
\hline 7 & 13.9 & 271.228 & 0.008923 & 1.6475 & 1.6999 & $\mathrm{M}-\mathrm{H}$ & $\mathrm{C}_{16} \mathrm{H}_{32} \mathrm{O}_{3}$ & 16-Hydroxypalmitate \\
\hline 8 & 14.78 & 297.2434 & 0.01004 & 1.8186 & 3.22444 & $\mathrm{M}-\mathrm{H}$ & $\mathrm{C}_{18} \mathrm{H}_{34} \mathrm{O}_{3}$ & 18-Hydroxyoleate \\
\hline 9 & 14.48 & 295.228 & 0.014379 & 1.7213 & 1.66762 & $\mathrm{M}-\mathrm{H}$ & $\mathrm{C}_{18} \mathrm{H}_{32} \mathrm{O}_{3}$ & 9(10)-EpOME \\
\hline 10 & 13.86 & 295.2284 & 0.00243 & 1.658 & 1.28566 & $\mathrm{M}-\mathrm{H}$ & $\mathrm{C}_{18} \mathrm{H}_{32} \mathrm{O}_{3}$ & 13-HODE \\
\hline 11 & 6.24 & 653.2632 & 0.012589 & 1.7245 & 4.57453 & $\mathrm{M}-\mathrm{H}$ & $\mathrm{C}_{36} \mathrm{H}_{38} \mathrm{~N}_{4} \mathrm{O}_{8}$ & Coproporphyrin III \\
\hline 12 & 18.58 & 279.2312 & 0.004448 & 1.5613 & 13.3247 & $\mathrm{M}-\mathrm{H}$ & $\mathrm{C}_{18} \mathrm{H}_{32} \mathrm{O}_{2}$ & Linoleate \\
\hline 13 & 20.9 & 283.2648 & 0.008692 & 1.5262 & 1.27355 & $\mathrm{M}-\mathrm{H}$ & $\mathrm{C}_{18} \mathrm{H}_{36} \mathrm{O}_{2}$ & Octadecanoic acid \\
\hline 14 & 7.83 & 201.1134 & 0.0214 & 1.6643 & 1.00239 & $\mathrm{M}-\mathrm{H}$ & $\mathrm{C}_{10} \mathrm{H}_{18} \mathrm{O}_{4}$ & Sebacic acid \\
\hline 15 & 19.37 & 317.2474 & 0.030716 & 0.58057 & 4.42563 & $\mathrm{M}+\mathrm{H}$ & $\mathrm{C}_{21} \mathrm{H}_{32} \mathrm{O}_{2}$ & $5 \alpha$-Pregnane-3,20-dione \\
\hline 16 & 4.17 & 265.1179 & 0.018487 & 0.40271 & 4.31748 & $\mathrm{M}+\mathrm{H}$ & $\mathrm{C}_{13} \mathrm{H}_{16} \mathrm{~N}_{2} \mathrm{O}_{4}$ & Phenylacetylglutamine \\
\hline 17 & 14.79 & 281.2474 & 0.015381 & 1.6879 & 2.37695 & $\mathrm{M}+\mathrm{H}$ & $\mathrm{C}_{18} \mathrm{H}_{32} \mathrm{O}_{2}$ & $\beta$-Linoleic acid \\
\hline 18 & 19.94 & 309.2791 & 0.003259 & 1.6437 & 2.33255 & $\mathrm{M}+\mathrm{H}$ & $\mathrm{C}_{20} \mathrm{H}_{36} \mathrm{O}_{2}$ & Eicosadienoic acid \\
\hline 19 & 20.45 & 401.3418 & 0.04793 & 0.63495 & 2.25178 & $\mathrm{M}+\mathrm{H}$ & $\mathrm{C}_{27} \mathrm{H}_{44} \mathrm{O}_{2}$ & 7-Ketocholesterol \\
\hline 20 & 4.18 & 134.0596 & 0.0004 & 0.31355 & 2.05813 & $\mathrm{M}+\mathrm{H}$ & $\mathrm{C}_{8} \mathrm{H}_{7} \mathrm{NO}$ & Indoxyl \\
\hline 21 & 21.67 & 297.2424 & 0.012856 & 1.5015 & 1.91806 & $\mathrm{M}+\mathrm{H}$ & $\mathrm{C}_{18} \mathrm{H}_{32} \mathrm{O}_{3}$ & 18-Oxooleate \\
\hline 22 & 20.72 & 315.2535 & 0.013926 & 1.8311 & 1.81478 & $\mathrm{M}+\mathrm{H}$ & $\mathrm{C}_{18} \mathrm{H}_{34} \mathrm{O}_{4}$ & (8E,10S)-10-Hydroperoxyoctadeca-8-enoate \\
\hline 23 & 13.36 & 287.2217 & 0.002671 & 1.9422 & 1.50515 & $\mathrm{M}+\mathrm{H}$ & $\mathrm{C}_{16} \mathrm{H}_{30} \mathrm{O}_{4}$ & Hexadecanedioate \\
\hline
\end{tabular}


TABle 2: Continued.

\begin{tabular}{|c|c|c|c|c|c|c|c|c|}
\hline Number & $\begin{array}{c}\mathrm{RT} \\
(\mathrm{min})\end{array}$ & $\mathrm{m} / z(\mathrm{Da})$ & $P$ value & FC & VIP & Adduct & Formula & Compound name \\
\hline 24 & 15.11 & 315.2539 & 0.001583 & 2.062 & 1.47203 & $\mathrm{M}+\mathrm{H}$ & $\mathrm{C}_{18} \mathrm{H}_{34} \mathrm{O}_{4}$ & 9,10-DHOME \\
\hline 25 & 15.53 & 297.2433 & 0.002945 & 1.6887 & 1.40775 & $\mathrm{M}+\mathrm{H}$ & $\mathrm{C}_{18} \mathrm{H}_{32} \mathrm{O}_{3}$ & 9(10)-EpOME \\
\hline 26 & 14.22 & 313.2379 & 0.010082 & 1.5503 & 1.36518 & $\mathrm{M}+\mathrm{H}$ & $\mathrm{C}_{18} \mathrm{H}_{32} \mathrm{O}_{4}$ & $\begin{array}{c}(9 \mathrm{Z}, 11 \mathrm{E})-(13 \mathrm{~S})-13 \text {-Hydroperoxyoctadeca-9,11- } \\
\text { dienoic acid }\end{array}$ \\
\hline 27 & 14.79 & 299.2581 & 0.005956 & 1.7695 & 1.301 & $\mathrm{M}+\mathrm{H}$ & $\mathrm{C}_{18} \mathrm{H}_{34} \mathrm{O}_{3}$ & cis-9,10-Epoxystearic acid \\
\hline 28 & 19.37 & 285.2216 & 0.031826 & 0.63012 & 1.23636 & $\mathrm{M}+\mathrm{H}$ & $\mathrm{C}_{20} \mathrm{H}_{28} \mathrm{O}$ & Retinal \\
\hline 29 & 13.86 & 297.2427 & 0.000278 & 1.7256 & 1.05818 & $\mathrm{M}+\mathrm{H}$ & $\mathrm{C}_{18} \mathrm{H}_{32} \mathrm{O}_{3}$ & 13-HODE \\
\hline 30 & 11.96 & 447.3107 & 0.022674 & 0.59386 & 1.04682 & $\mathrm{M}+\mathrm{H}$ & $\mathrm{C}_{27} \mathrm{H}_{42} \mathrm{O}_{5}$ & 3-Dehydro-2-deoxyecdysone \\
\hline 31 & 6.18 & 160.0756 & 0.001919 & 0.5921 & 1.04347 & $\mathrm{M}+\mathrm{H}$ & $\mathrm{C}_{10} \mathrm{H}_{9} \mathrm{NO}$ & Indole-3-acetaldehyde \\
\hline 32 & 15.53 & 319.2255 & 0.007767 & 1.7745 & 1.02064 & $\mathrm{M}+\mathrm{H}$ & $\mathrm{C}_{20} \mathrm{H}_{30} \mathrm{O}_{3}$ & Leukotriene A4 \\
\hline 33 & 13.9 & 255.2319 & 0.003714 & 1.6028 & 0.993851 & $\mathrm{M}+\mathrm{H}$ & $\mathrm{C}_{16} \mathrm{H}_{30} \mathrm{O}_{2}$ & (9Z)-Hexadecenoic acid \\
\hline 34 & 12.57 & 267.1955 & 0.006426 & 1.7148 & 0.882565 & $\mathrm{M}+\mathrm{H}$ & $\mathrm{C}_{16} \mathrm{H}_{26} \mathrm{O}_{3}$ & Tetranor 12-HETE \\
\hline 35 & 17.74 & 451.3423 & 0.016417 & 1.5795 & 0.807997 & $\mathrm{M}+\mathrm{H}$ & $\mathrm{C}_{27} \mathrm{H}_{46} \mathrm{O}_{5}$ & $\begin{array}{l}\text { 3alpha,7alpha,12alpha-Trihydroxy-5beta- } \\
\text { cholestanoate }\end{array}$ \\
\hline 36 & 8.72 & 191.1065 & 0.02261 & 1.7698 & 0.675408 & $\mathrm{M}+\mathrm{H}$ & $\mathrm{C}_{12} \mathrm{H}_{14} \mathrm{O}_{2}$ & Propyl cinnamate \\
\hline 37 & 17.86 & 317.2692 & 0.016669 & 1.5145 & 0.667866 & $\mathrm{M}+\mathrm{H}$ & $\mathrm{C}_{18} \mathrm{H}_{36} \mathrm{O}_{4}$ & 9,10-Dihydroxystearate \\
\hline 38 & 13.36 & 223.2056 & 0.001659 & 1.6801 & 0.580941 & $\mathrm{M}+\mathrm{H}$ & $\mathrm{C}_{15} \mathrm{H}_{26} \mathrm{O}$ & Farnesol \\
\hline
\end{tabular}

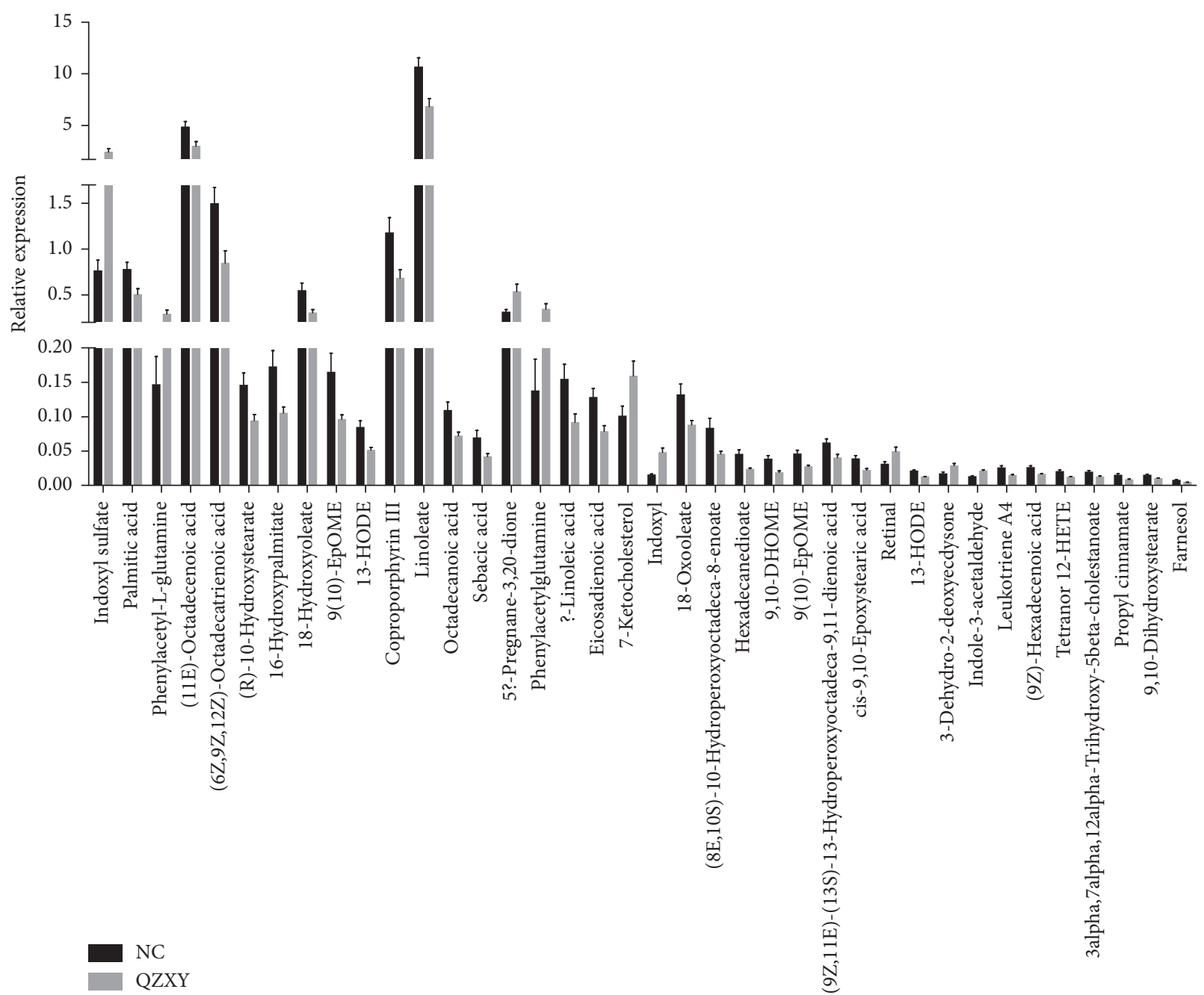

FIGURE 7: Comparison of different metabolite intensities and specific degrees of differences between QZXY and control groups. 
TABle 3: A total of 11 differential metabolic pathways were identified in this study.

\begin{tabular}{|c|c|c|c|c|c|c|c|}
\hline Pathway name & Match status & $P$ & $-\log (p)$ & Holm $\mathrm{p}$ & FDR & Impact & Details \\
\hline Linoleic acid metabolism & $3 / 5$ & $3.2013 E-5$ & 10.349 & 0.0026891 & 0.0026891 & 1.0 & KEGG SMP \\
\hline Biosynthesis of unsaturated fatty acids & $4 / 36$ & 0.0018721 & 6.2807 & 0.15538 & 0.078626 & 0.0 & KEGG \\
\hline Retinol metabolism & $1 / 17$ & 0.23408 & 1.4521 & 1.0 & 1.0 & 0.24227 & KEGG SMP \\
\hline Porphyrin and chlorophyll metabolism & $1 / 30$ & 0.37663 & 0.97648 & 1.0 & 1.0 & 0.02955 & KEGG \\
\hline Arachidonic acid metabolism & $1 / 36$ & 0.4335 & 0.83587 & 1.0 & 1.0 & 0.07615 & KEGG SMP \\
\hline Fatty acid elongation & $1 / 39$ & 0.46003 & 0.77646 & 1.0 & 1.0 & 0.0 & KEGG SMP \\
\hline Fatty acid degradation & $1 / 39$ & 0.46003 & 0.77646 & 1.0 & 1.0 & 0.0 & KEGG SMP \\
\hline N-Glycan biosynthesis & $1 / 41$ & 0.47705 & 0.74013 & 1.0 & 1.0 & 0.0 & KEGG \\
\hline Tryptophan metabolism & $1 / 41$ & 0.47705 & 0.74013 & 1.0 & 1.0 & 0.0139 & KEGG SMP \\
\hline Primary bile acid biosynthesis & $1 / 46$ & 0.51739 & 0.65896 & 1.0 & 1.0 & 0.03267 & KEGG SMP \\
\hline Fatty acid biosynthesis & $1 / 47$ & 0.52509 & 0.64419 & 1.0 & 1.0 & 0.01473 & KEGG SMP \\
\hline
\end{tabular}

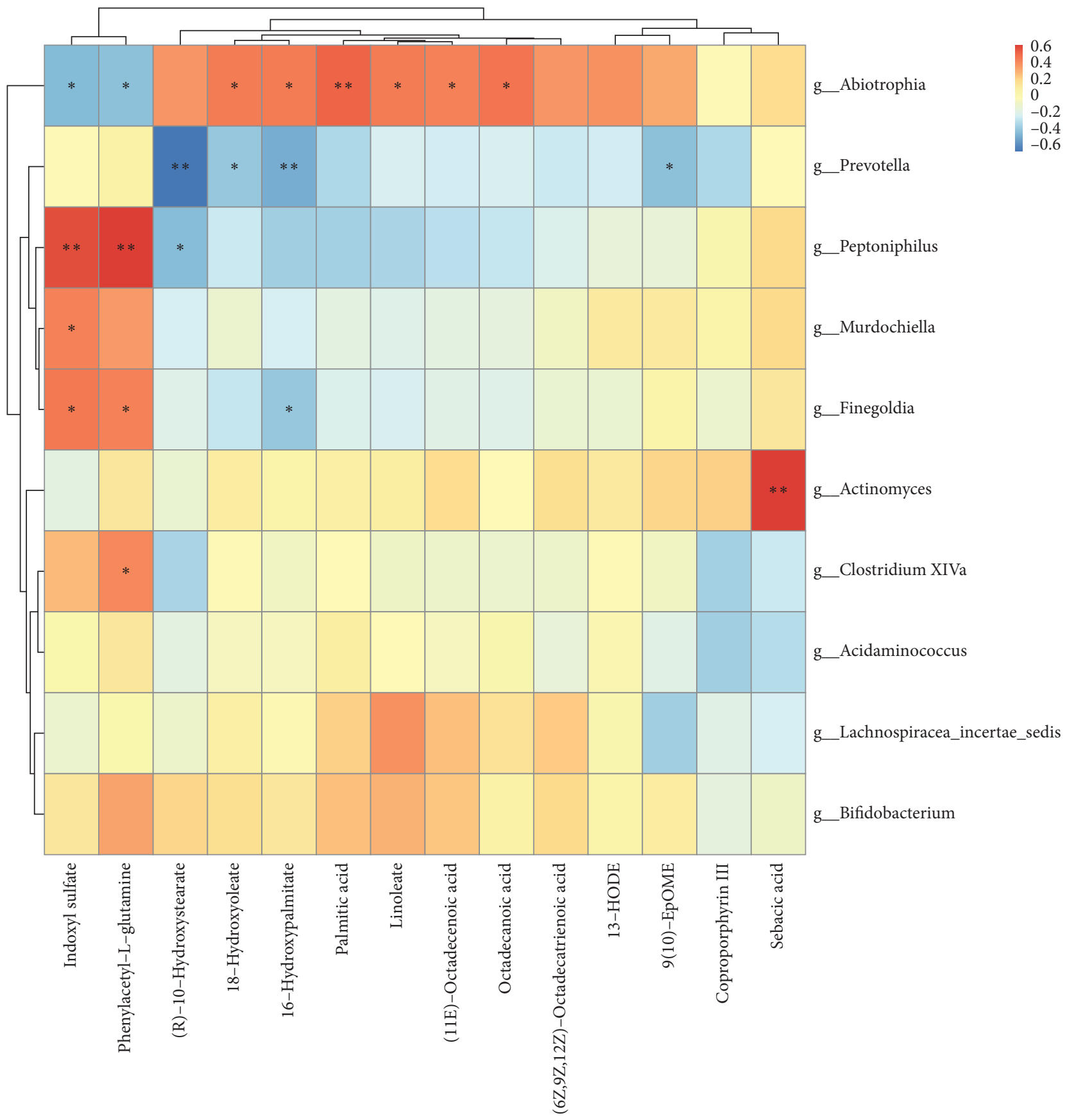

FIGURE 8: Correlation between anion metabolites and differential flora. 


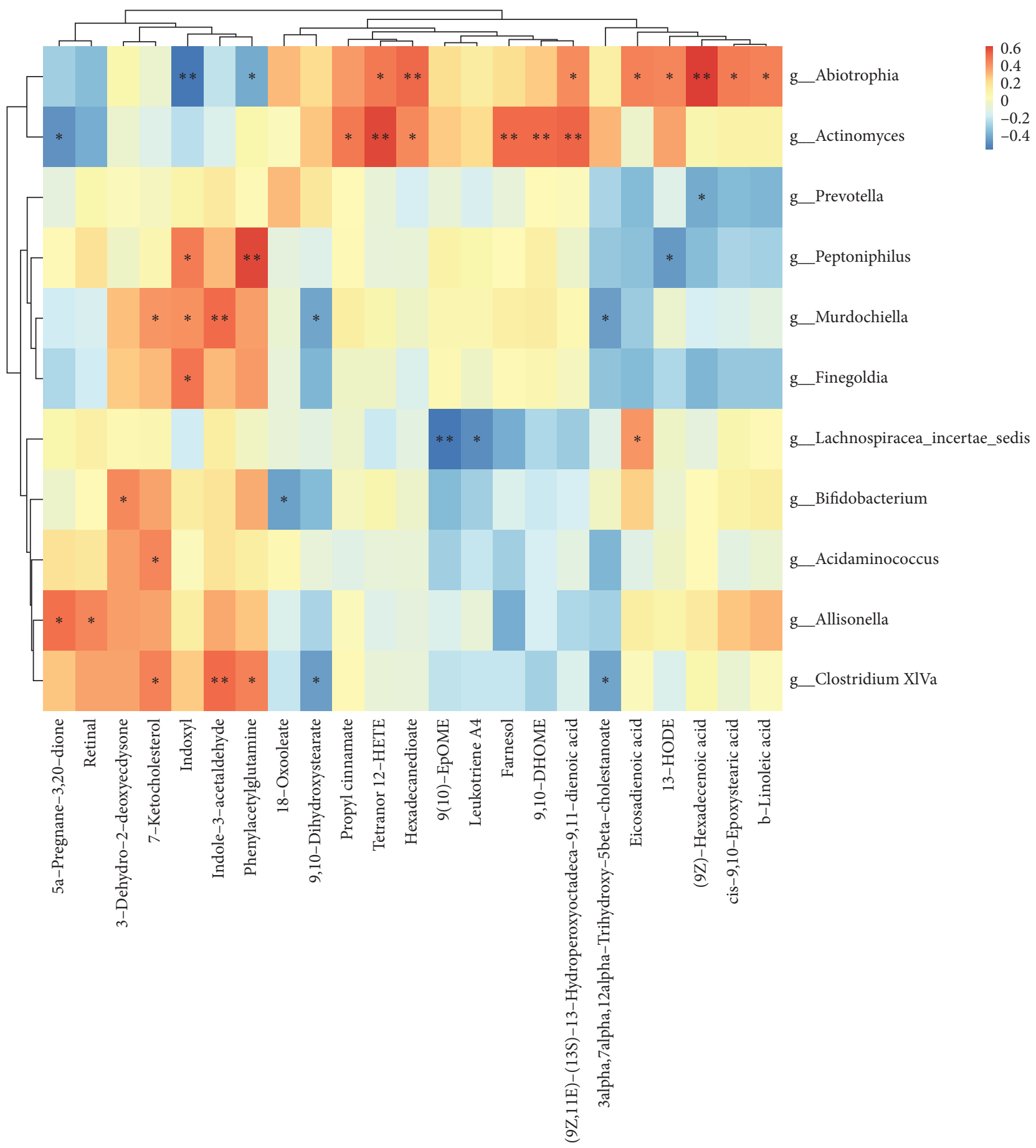

Figure 9: Correlation between positive ion metabolites and differential flora.

[13]. Indophenol can be produced by isopentenylation of tryptophan derivatives. Tryptophan has been found to be metabolized rapidly in many tumors. Peptoneophilus is positively correlated with metabolites, such as glutamine and indophenol. It has been found to be enriched in endometrial carcinoma tissues [14]. No correlation has been found between peptones and lung cancer. This suggests that peptones may affect the formation and development of lung cancer by regulating a variety of metabolic pathways. Prevotella and Bifidobacteria were negatively correlated with lipid metabolites (palmitic acid, oleate ester). Palmitic acid can promote the epithelial-mesenchymal transformation of lung tumor cells and thus enhance their invasion. Microflora has been shown to be involved in host regulation of metabolism and in the production of hormones and bile acids [15]. The present study found that many bacterial genera were negatively correlated with lipid metabolism. This suggested that there may be a certain mechanism between bacterial flora and lipid metabolism to regulate the occurrence and development of lung cancer. NSCLC patients with QZXY 


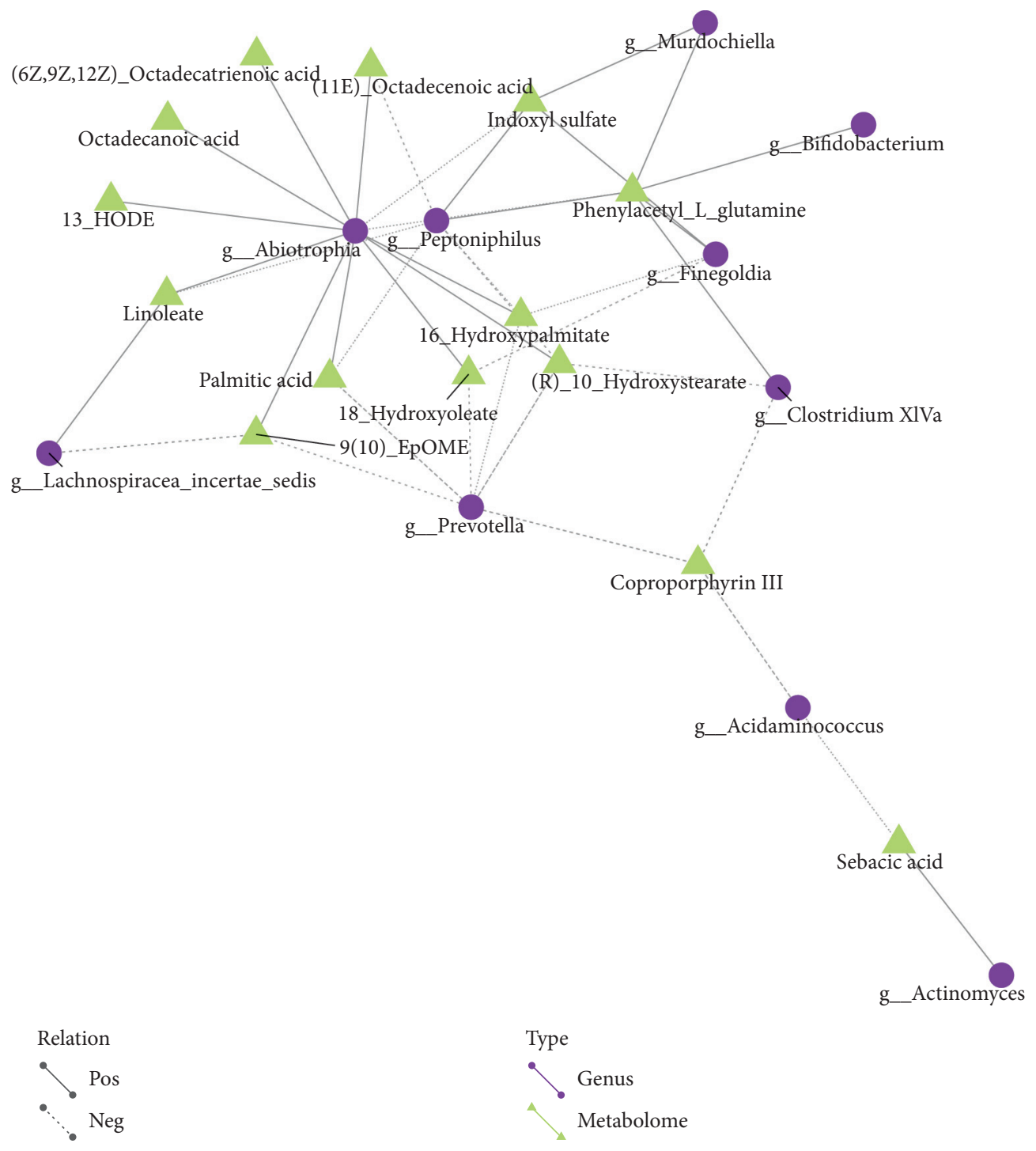

Figure 10: Results of differential metabolite and flora network regulation analysis in anion mode show that Abiotrophia and Peptoniphilus are closely related to many metabolites. This suggests that they may be the key bacteria in the development of lung cancer.

syndrome often exhibit blood stasis signs, such as stasis spots on the tip of the tongue. Studies [16] have shown that application of Danshensu, an extract of Salvia miltiorrhiza with the effect of promoting blood circulation and removing blood stasis, can effectively regulate dyslipidemia in hyperlipidemia rats, providing a theoretical basis for the correlation between abnormal lipid metabolism and QZXY syndrome.

The present study explored the changes in signaling pathways in NSCLC patients with QZXY at the multiomics level. Five signaling pathways, including MAPK, PI3KAKT, Ras, cancer pathways, and cytokine-cytokine receptor interaction, were screened using the linked data network diagram. As a traditional Chinese medicine for promoting blood circulation and removing blood stasis, the active ingredients in Panax notoginseng can act on EGFR, MAPK14, and other targets, regulating cancer pathways, cytokine-cytokine receptor interactions, and other signaling pathways and exert anti-inflammatory effects [17]. It was also found that corydus can exert the effect of promoting blood circulation by regulating the cytokine-cytokine receptor interaction and other signaling pathways [18]. The combination of Astragalus and Zedoary (toning Qi and activating blood circulation) can downregulate the expression of MAPK and inhibit the growth and metastasis of transplanted Lewis lung cancer tumors in tumor-bearing mice [19]. To summarize, we speculated that the above five pathways were closely related to lung cancer. Further studies may help to explore how to use TCM theories combined with biomarkers to deeply explore the mechanism of TCM treatment for lung cancer, which is particularly important and can provide new targets for clinical treatment.

We also attempted to analyze the metabolomic differences between the QZXY and nQZXY groups. Four metabolites with ROC $>0.9$ and high sensitivity and specificity were found. Pseudouridine is a component of tRNA synthesized by the human body, which can significantly reduce 


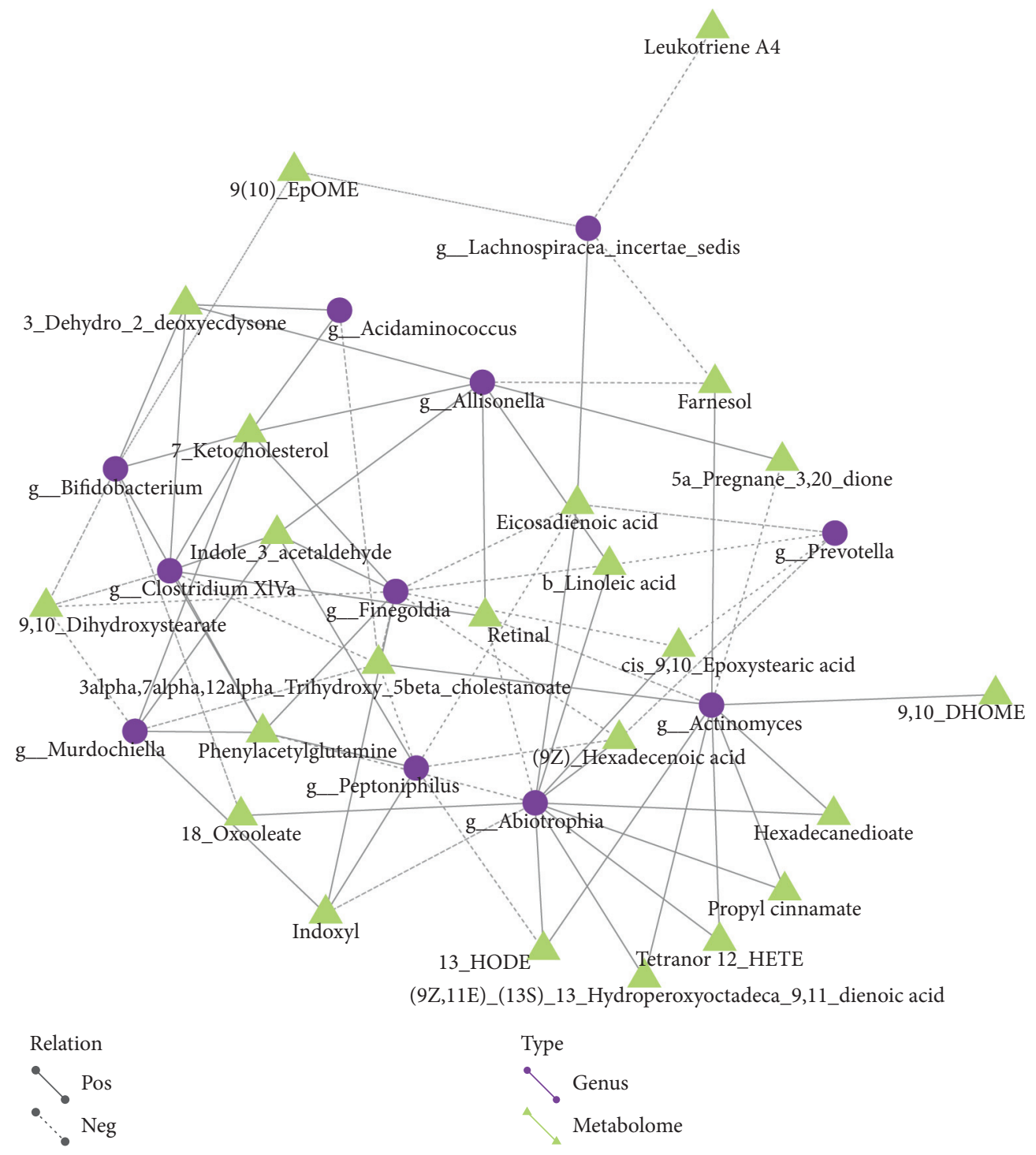

FIgURE 11: Results of differential metabolite and flora network regulation analysis in positive mode show that Clostridium XIVa, Aclinomyces, Finegoldia, Abiotrophia, and Peptoniphilus are closely related to many metabolites. This suggests that they also may be the key bacteria in the development of lung cancer.

the ability of RNA to stimulate the immune system. It may help lung cancer cells to avoid an immune response. L-Glutamine has also been analyzed. The study results indicated that compared to NSCLC patients with other syndromes, there were significant differences in metabolites in patients with QZXY syndrome. This meant that QZXY syndrome could be diagnosed and differentiated from other syndromes, suggesting that there was microscopic evidence of its presence. However, the sample size in the present study was small, potentially creating a source of bias. Therefore, these results only provided a limited reference value for future investigations. One prior study [20] has found that the syndromic types had different effects on tumor progression by establishing the "Zheng-first" and "Tumor-first" models of subcutaneous pancreatic cancer in mice with different syndromic types. These results may provide a direction for follow-up studies.

The study of TCM syndromes using systems biology methods is helpful to provide an objective basis for syndrome differentiation and treatment and to suggest highly effective and low-toxicity individualized treatment for tumor patients. In this study, combined multiomics analysis was conducted in NSCLC patients with QZXY. This investigation not only promoted the study of lung cancer mechanisms and determine its pathogenic targets, but also provided theoretical support and technical foundation for the prevention and treatment of lung cancer using traditional Chinese medicine. 


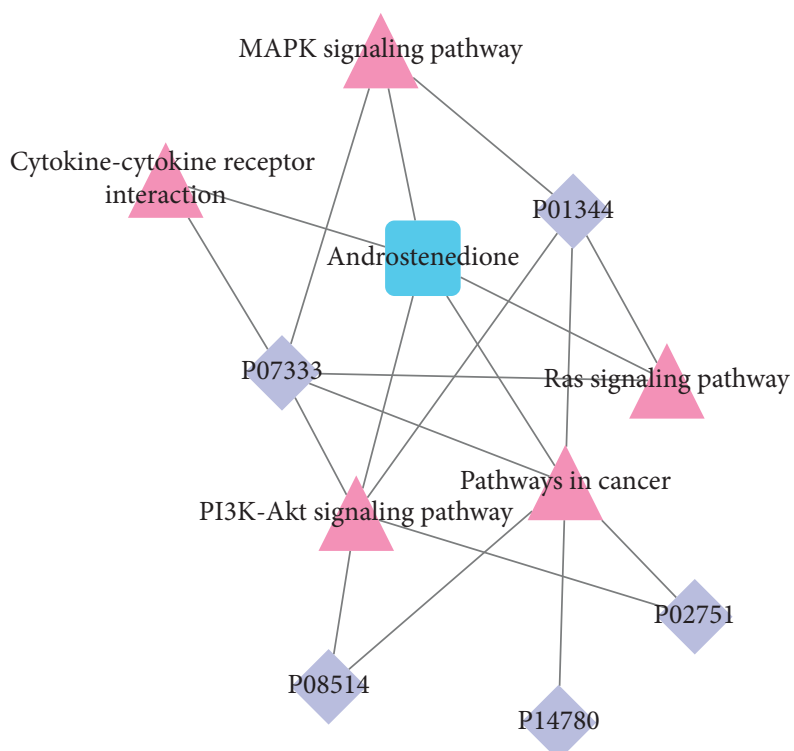

FIGURE 12: The patient-based differential proteins IGF2, CSF1R, ITA2B, MMP9, FINC, and the downstream metabolite androstenedione were associated with MAPK, PI3K/Akt, Ras signaling pathway, while cancer pathways and cytokine-cytokine receptor interactions were correlated.

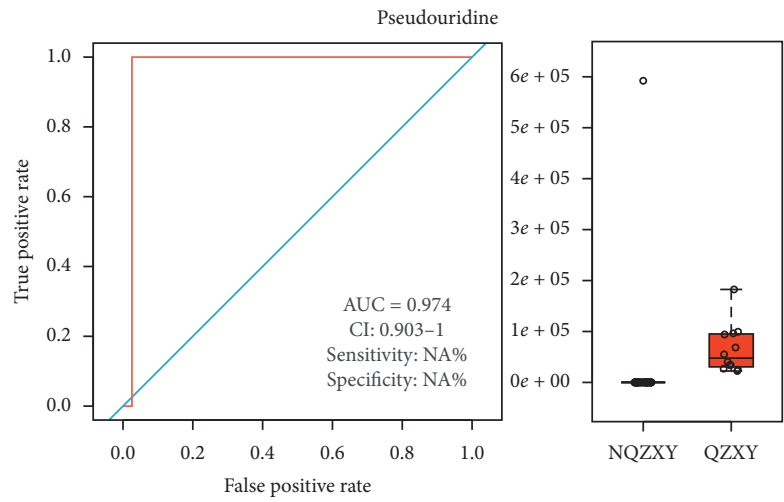

(a)

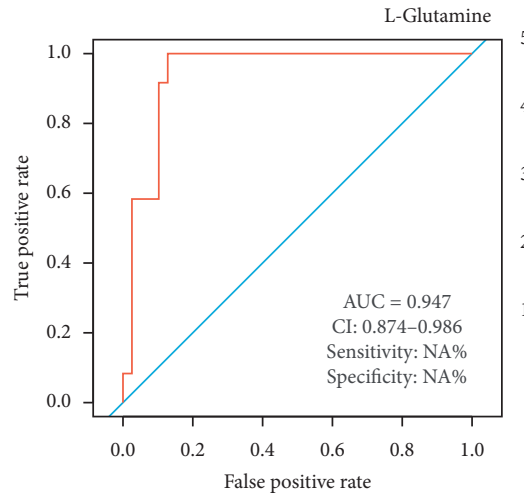

(c)

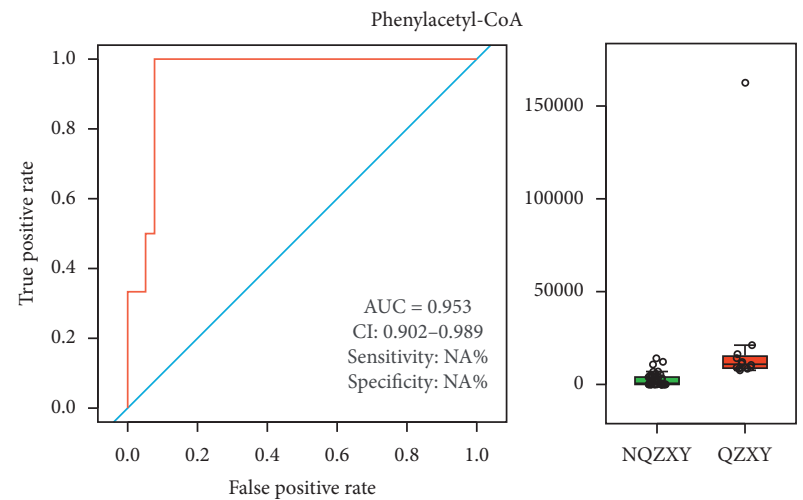

(b)

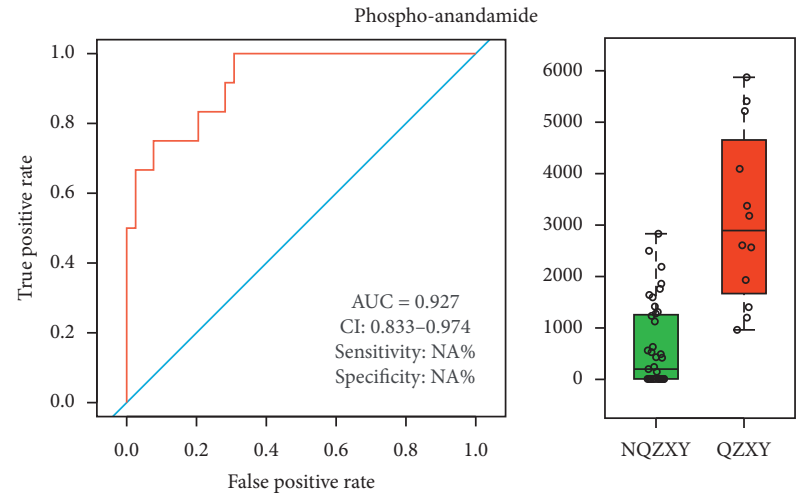

(d)

Figure 13: Pseudouridine, phenlacetyl-CoA, L-glutamine, and phospho-anandamide had the highest area under the curve for identification of the two, where their sensitivity was $97.4 \%, 92.3 \%, 98.9 \%$, and $92.3 \%$ and specificity was $100 \%, 100 \%, 100 \%$, and $75 \%$, respectively. The AUC values for other metabolites were all <0.9. (a) Pseudoridine. (b) Phenylacetyl-CoA. (c) L-Glutamine. (d) Phospho-anandamide. 


\section{Conclusions}

NSCLC with Qi stagnation and blood stasis syndrome had specific intestinal flora and protein and metabolites, which were closely related to the occurrence and development of tumors.

\section{Data Availability}

The data used to support the findings of this study are available from the author upon request.

\section{Conflicts of Interest}

The authors declare no competing interests.

\section{Acknowledgments}

This study was supported by National Natural Science Foundation of China, Study on the Immune Regulation Effect of Intestinal Akk Bacteria in Lung Cancer Mice and the Intervention of Qingfei Mixture Based on the Theory of "Lung and Large Intestine Surface and Inside" (no. 81973771), Zhejiang Provincial Traditional Chinese Medicine Science and Technology Plan Project: Study on the Correlation between TCM Syndromes and Modern Biomarkers in Patients with Non-Small Cell Lung Cancer Based on Intestinal Microflora Structure Characteristics and Metabolomics (no. 2019ZZ002), and Affilliation: Institute of Basic Medicine and Cancer, Chinese Academy of Sciences, Hangzhou 310022, China.

\section{References}

[1] X. Xl, Y. JiangL. P. Shen et al., Analysis of survival of 600 elderly patients with non-small cell lung cancer treated with traditional Chinese medicine," Journal of Traditional Chinese Medicine, vol. 60, no. 1, pp. 31-35, 2019.

[2] X. Y. Mu, H. R. Bai, X. H. Zhou et al., "JinFuKang oral liquid combined with Jian Tang combined with chemotherapy in the treatment of III B-stage non-small cell lung cancer with deficiency of Qi and Yin," Clinical Research in China, vol. 32, no. 9, pp. 1271-1274, 2019.

[3] C. H. Wang, "The efficacy and safety of traditional Chinese medicine combined with gefitinib in the treatment of advanced non-small cell lung cancer," Traditional Chinese Medicine, vol. 42, no. 8, pp. 1937-1945, 2019.

[4] J. H. LI and T. Li, "Clinical study of Invigorate the circulation of blood decoction combined with cisplatin for advanced nonsmall cell lung cancer," Journal of Traditional Chinese Medicine, vol. 32, no. 1, pp. 13-17, 2017.

[5] X. Qian, Z. Chen, and A. Q. Zhang, "Intestinal microbiota and immune function in patients with non-small cell lung cancer," Zhejiang Journal of Traditional Chinese Medicine, vol. 55, no. 9, pp. 625-627, 2020.

[6] D. D. Li, P. M. Liu, and X. Q. Zhang, "Professor Liu Peimin's clinical experience of XuefuZhuyu Decoction in the treatment of lung cancer with qi stagnation and blood stasis," AsiaPacific Traditional Medicine, vol. 13, no. 14, pp. 91-92, 2017.

[7] J. Li, Chinese Society of Clinical Oncology (CSCO) Guidelines for the Diagnosis and Treatment of Primary Lung Cancer, People's Medical Publishing House, Beijing, China, 2019.
[8] X. Chen, X. D. Wang, and P. T. Zhang, "Traditional Chinese medicines in the management of cardiovascular diseases: a comprehensive systematic review," Chinese Journal of Traditional Chinese Medicine, vol. 32, no. 12, pp. 5505-5509, 2017.

[9] Z. Y. Wang, X. Qian, and A. Q. Zhang, "Intestinal microflora diversity in patients with lung adenocarcinoma based on the theory of lung and colon surface and interior," Zhejiang Journal of Traditional Chinese Medicine, vol. 54, no. 10, pp. 710-711, 2019.

[10] N. Shaverdian, D. Veruttipong, J. Wang, P. Kupelian, M. Steinberg, and P. Lee, "Location matters: stage I nonsmall-cell carcinomas of the lower lobes treated with stereotactic body radiation therapy are associated with poor outcomes," Clinical Lung Cancer, vol. 18, no. 2, pp. e137-e142, 2017.

[11] L. Zhao, J. Wang, H. Li et al., "Safety and efficacy of tianfoshen oral liquid in non-small cell lung cancer patients as an adjuvant therapy," Evidence-Based Complementary and Alternative Medicine, vol. 2019, Article ID 1375439, 2019.

[12] J. Yang, X. Zhu, P. Yuan, J. Liu, B. Wang, and G. Wang, "Efficacy of traditional Chinese Medicine combined with chemotherapy in patients with non-small cell lung cancer (NSCLC): a meta-analysis of randomized clinical trials," Supportive Care in Cancer, vol. 28, no. 8, pp. 3571-3579, 2020.

[13] Y. Lai, Effects of Glutamine on Proliferation, Migration and Expression of TNF- $\alpha$ and NF-Kb P65 in Human Lung Adenocarcinoma A549 Cells, Southwest Medical University, Luzhou, China, 2018.

[14] M. R. Walther-António, J. Chen, F. Multinu et al., "Potential contribution of the uterine microbiome in the development of endometrial cancer," Genome Medicine, vol. 122, no. 1, p. 8, 2016.

[15] Q. Mao, F. Jiang, R. Yin et al., "Interplay between the lung microbiome and lung cancer," Cancer Letters, vol. 40, 2017.

[16] B. L. Chang, H. Guo, F. Yuan et al., "Effects of yiqi dehumidification and huoxue decoction on sugar and lipid metabolism in obese rats," Hebei Journal of Traditional Chinese Medicine, vol. 35, no. 1, pp. 1-3+32, 2020.

[17] H. T. Pang, D. S. Luo, and J. Guo, "The extraordinary transformation of traditional Chinese medicine: processing with liquid excipients," Journal of Traditional Chinese and Herbal Medicine, vol. 51, no. 21, pp. 5538-5547, 2020.

[18] T. J. Zhang, J. Xu, Y. Q. Han et al., "Study on Q-marker of traditional Chinese medicine: quality evaluation and quality standard of Corydalis corydalis," Chinese Herbal Medicine, vol. 47, no. 9, pp. 1458-1467, 2016.

[19] C. Y. Xu, Summary of Professor Dou Yongqi's Academic Thoughts on the Treatment Of Cancer And Study on the Antitumor Angiogenesis Mechanism of Astragalus Zedoary Compatibility, Medical College of Chinese People's Liberation Army, Beijing, China, 2018.

[20] F.-J. Wang, P. Wang, L.-Y. Chen et al., "TAM infiltration differences in "Tumor-First" and "ZHENG-First" models and the underlying inflammatory molecular mechanism in pancreatic cancer," Integrative Cancer Therapies, vol. 17, no. 3, pp. 707-716, 2018. 